\title{
Modelado de la gestión curricular basada en procesos de negocio sensibles al contexto*
}

\author{
Curriculum management modeling based on context-sensitive business \\ processes
}

\author{
Jorge E. Giraldo-Plaza \\ PhD en Ingeniería, Politécnico Colombiano Jaime Isaza Cadavid, \\ Medellín, Colombia, jegiraldo@elpoli.edu.co \\ Demetrio A. Ovalle-Carranza \\ PhD en Informática, Universidad Nacional de Colombia, \\ Medellín, Colombia, dovalle@unal.edu.co
}

\begin{abstract}
Cómo citar / How to cite
Giraldo-Plaza, J. E.; Ovalle-Carranza, D. A. (2020). Modelado de la gestión curricular basada en procesos de negocio sensibles al contexto. Revista CEA, v. 6, n. 12, 129-146. https://doi.org/10.22430/24223182.1526
\end{abstract}

Recibido: 7 de febrero de 2020

Aceptado: 17 de abril de 2020

\section{Resumen}

El objetivo de este artículo es presentar una aproximación del modelado de la gestión curricular en instituciones universitarias a través de los fundamentos de los procesos de negocio con especial enfoque en la información sensible al contexto. La importancia del contexto se refleja en la necesidad de controlar mayor información del ambiente que permita incrementar la expresividad en la representación y razonamiento inteligente sobre el conocimiento. El contexto se modela teniendo en cuenta entidades propias del dominio de ejecución y situaciones contextuales asociadas a dicho entorno de ejecución. El razonamiento automático se realiza aplicando un algoritmo inteligente que emplea técnicas de agrupamiento, el cual tiene en cuenta información contextual del proceso y genera alertas contextuales que apoyen la toma de decisiones. Para el procesamiento automático se diseñó un sistema de simulación y registro de ejecuciones de procesos, representadas en conjuntos de datos para la bitácora de cada proceso. Dentro de los resultados obtenidos, se tiene una representación formal de la gestión curricular a través de situaciones, que enriquecen la información de cada uno de los procesos de negocio. Se puede concluir que la información relacionada con el currículo es susceptible al contexto y por tanto puede afectar la ejecución de los procesos asociados

\footnotetext{
* Este artículo se deriva del proyecto titulado «Método de integración inteligente de procesos de negocio sensible y adaptado al contexto» y está parcialmente financiada por la Universidad Nacional de Colombia a través del programa de apoyos a posgrados, asociado al estudiante de doctorado Jorge E. Giraldo Plaza con código Quipu 200000013723.
} 
a la gestión curricular. De esta forma, se recomienda diseñar reglas de razonamiento automático que permitan tener en cuenta el contexto para la toma de decisiones en procesos de gestión curricular.

Palabras clave: gestión curricular universitaria, técnicas de modelado, gestión de procesos de negocio.

\title{
Clasificación JEL: O31.
}

\begin{abstract}
This paper aims to propose an approach to curriculum management modeling in universities based on the fundamentals of business processes and with a special focus on context-sensitive information. The importance of the context lies in the need to control more information of the surroundings to improve expressiveness in the representation and intelligent reasoning of knowledge. The context is modeled by entities related to the domain of execution, as well as by contextual situations associated with said environment. Automated reasoning is carried out by implementing an intelligent algorithm that uses clustering techniques. This algorithm considers the contextual information of a process and then generates contextual alerts that support decision making. For the automated processing of such information, we design a system that simulates and records process executions using data sets for the log of each process. Some of our findings include a formal representation of curriculum management based on situations that enrich the information of each business process. According to the results, we may conclude that curriculum-related information is context-sensitive and might, thus, affect the execution of the processes associated with curriculum management. Therefore, we recommend designing automated reasoning rules that consider the context for decision making in curriculum management processes.
\end{abstract}

Keywords: University curriculum management, modeling techniques, business process management.

\section{JEL classification: 031.}

\section{INTRODUCCIÓN}

La gestión curricular está encargada de la definición de procesos y prácticas educativas que realizan diversos actores en torno a un currículo en una institución de formación con el fin de promover ambientes de reflexión, construcción y mejoramiento del mismo (González, 2015; Morales Martínez et al., 2017). Es importante aclarar que la gestión curricular comprende políticas y procedimientos que permiten planificar, diseñar, evaluar, analizar y rediseñar todos los aspectos de un currículo con el fin de lograr un mejoramiento continuo, orientado a ser pertinente a la comunidad que este impacta (Maia \& Pacheco, 2019).

En este contexto, se han propuesto nuevas estructuras de cambio en la gestión curricular a partir de teorías psicológicas de aprendizaje (Watson et al., 2007; Viciana \& Mayorga-Vega, 2016) y de tomar en cuenta el papel de los actores para la dinamización constante de los currículos (Barrón Tirado \& Díaz Barriga, 2016). Adicionalmente, se ha profundizado sobre las tareas necesarias para la estructuración de sistemas de gestión del currículo de manera que puedan estructurarse de forma 
autónoma y participativa (Kim, 2005). Desde esta perspectiva, existen herramientas digitales de gestión curricular orientadas a una articulación más estructurada de los factores y componentes de estos procesos (Cottrell et al., 2004).

Asimismo, en áreas del conocimiento cuyos contenidos curriculares son diversos y cambiantes, como es el caso de los programas en el área de la salud, se hace necesario plantear dinámicas de seguimiento curricular que permitan servir de soporte a procesos de actualización de contenidos y tengan en cuenta los cambios del contexto en aspectos económicos, sociales y culturales (Jacobs et al., 2005; Watson et al., 2007).

Salgado Bustamante y Medel Toro (2015) plantean que la gestión del currículo se compone de los siguientes factores: (1) la gestión pedagógica, que se encarga de la organización, planeación, monitoreo y evaluación de una propuesta curricular, (2) la aplicación de procesos de enseñanzaaprendizaje, que implica un correcto uso de las estrategias de aprendizaje definidas, (3) el desarrollo del estudiante, donde se garantiza un desarrollo emocional, social y académico apropiado para cada uno de los estudiantes y (4) el entorno de ejecución. Todo ello permite que los procesos curriculares sean sensibles a la información que se procesa dentro de su ambiente de ejecución.

Por su parte, los procesos de negocio (BP: Business Process) pueden ser modelados teniendo en cuenta la información contextual asociada al proceso, la cual puede ser aprovechada para realizar razonamiento inteligente sobre ellos (Ploesser et al., 2009). Así mismo, los procesos de negocio sensibles al contexto buscan procesar información de entidades y situaciones contextuales relacionadas con el dominio y ambiente de ejecución del proceso. Con el fin de mejorar el razonamiento del proceso, es posible modelar la información contextual dentro de una institución educativa y sus procesos asociados.

Entre de las investigaciones relacionadas con BPM (Business Process Management) y gestión curricular, Svensson y Hvolby (2012) proponen un modelo de referencia basado en la ejecución de procesos de negocio para la gestión universitaria. Su principal aporte está en generar métricas de desempeño para procesos claves como la admisión universitaria y el pago de matrícula. En esa misma línea, Kahloun y Channouchi (2016) proponen un conjunto de métricas de evaluación de modelos de proceso en ambientes universitarios, por ejemplo, la definición de cupos para un curso. También Strîmbei et al., (2016) proponen una arquitectura de referencia empresarial basada en procesos, para ser aplicada en ambientes universitarios, específicamente en administración de infraestructura tecnológica.

Saidani y Nurcan (2009) identifican las variables del proceso que pueden ser afectadas por el contexto. Se presenta una jerarquización del contexto basado en cuatro componentes, a saber, el tiempo, la ubicación, los recursos y la organización. Rosa et al., (2015) proponen un sistema sensible al contexto para la evaluación de competencias universitarias, tomando como contexto las variables relacionadas con el tiempo dentro del proceso. Giraldo Plaza et al., (2019) modelan un proceso de admisión universitaria sensible al contexto, cuyas variables consideradas son ubicación geográfica y documentación.

Con base en lo anterior, se evidencia un gran potencial para la modelación de la gestión curricular desde un punto de vista de procesos de negocio. Por ello, el objetivo de este artículo es presentar un 
modelado de la gestión curricular a través de procesos de negocio sensibles al contexto. Previamente validada, dicha información contextual mejora el razonamiento y garantiza la adaptación de los procesos relacionados con la gestión curricular con base en situaciones específicas del contexto. El resto del documento presenta el modelado de los procesos de negocio identificados en la gestión curricular, así como la propuesta de un modelo de contexto. Luego, se describe el diseño e implementación del mecanismo de razonamiento que facilita la adaptación del proceso. Por último, se presenta el análisis de resultados, conclusiones y trabajo futuro.

\section{MARCO TEÓRICO}

Un proceso de negocio, según Weske (2019), se define como un conjunto de actividades ejecutadas de manera lógica sobre un ambiente técnico y organizacional para llevar a cabo un objetivo de negocio (por ejemplo, proceso de matrícula). Un BP puede ser descrito por medio de un modelo de proceso, el cual es la descripción formal del proceso de negocio, en donde se incluye sus actividades, actores y flujo de trabajo (Benedict et al., 2013).

Dentro del marco de la gestión curricular, el currículo es visto como el conjunto de criterios, métricas, planes de estudio, programas académicos, metodologías y procesos que permiten la formación integral y la construcción de una identidad cultural (Decreto 230 de 2002). El currículo incluye los recursos académicos, humanos y físicos para poner en práctica las políticas institucionales y el Proyecto Educativo Institucional (Makura \& Makura, 2012).

Cabe señalar, que el currículo genera un tejido social a partir de la conexión de la sociedad y la institución educativa. Así mismo, plantea los lineamientos que amparan un proyecto educativo institucional y agrupa todas aquellas prácticas pedagógicas y técnicas de enseñanza-aprendizaje que garanticen la adquisición de conocimientos generales y específicos (Zimmer \& Keiper, 2020).

Según Dey (2001), se define el contexto como "cualquier información que puede ser usada para caracterizar la situación de una entidad. Una entidad es una persona, lugar u objeto que se considera relevante en la interacción entre el usuario y la aplicación, incluyendo al usuario y a la aplicación misma". La Computación Sensible al Contexto tiene como objetivo principal el uso del contexto para el mejoramiento de la interacción entre computadores y usuarios. La sensibilidad al contexto radica en la capacidad de adaptarse a situaciones contextuales; por tanto, un sistema sensible al contexto es aquel que tiene la capacidad de comprender el ambiente en el cual es ejecutado y adaptar sus operaciones para proveer la mejor experiencia al usuario (Krumm, 2010).

\section{METODOLOGÍA}

El tipo de investigación se estructura desde un enfoque aplicado, pues busca la manera de alinear los conceptos de la gestión curricular, los procesos de negocio y la computación orientada al contexto con el fin de lograr currículos adaptables a las situaciones. La investigación es proyectiva, pues busca validar dichos conceptos por medio de la definición, construcción e implementación de casos de estudio que permiten la definición de proyectos.

Frente al diseño del proceso de investigación, se plantean las siguientes fases estratégicas: 
Fase 1. Definición de arquitectura de la gestión curricular basada en procesos de negocio.

En esta fase se toma como referente el ciclo de gestión de los procesos de negocio propuesto por Dumas et al., (2013). Allí se plantea la identificación de los procesos relacionados con la gestión curricular, la cual consta de la definición de actividades de negocio asociadas, la identificación de roles y su relación con las actividades y la definición de una arquitectura de procesos para la gestión curricular. Los actores identificados para cada uno de los procesos son: Estudiante (E), Docente (D), Directivos (Dr), Sector (S), Organismos disciplinares (O) y Comunidad (C). Cada uno de ellos brinda información al proceso que puede afectar su contexto y por lo tanto las decisiones que puedan tomarse durante la ejecución del proceso. Este insumo es utilizado para proponer la arquitectura de procesos destinada a la adopción de la gestión curricular.

\section{Fase 2. Propuesta de un modelo de contexto para los procesos de la gestión curricular.}

En esta fase las entidades definidas para el contexto de la gestión curricular son: recursos asociados, estructura del proceso, comportamiento del proceso, roles, organización y dominio. El valor contextual debe considerar la información dispuesta en cada uno de los procesos y sus objetos. Así mismo, las situaciones contextuales se definen teniendo en cuenta los elementos contextuales y sus valores. Para esto se diseña un esquema de representación de procesos de negocio mediante sintaxis XPDL (XML Process Definition Language), con el propósito de asociar restricciones para su posterior procesamiento en un mecanismo de inferencia.

\section{Fase 3. Diseño del mecanismo de razonamiento sobre procesos de negocio sensibles al contexto.}

En esta fase se plantea el diseño del sistema inteligente encargado del procesamiento de la información relacionada con los procesos, el procesamiento del contexto y el razonamiento de este. Lo anterior se convierte en el insumo para la toma decisiones frente al comportamiento de las variantes del proceso (La Rosa et al., 2017). El diseño presentado comprende un sistema de representación de modelos de procesos, un sistema de razonamiento y una arquitectura tecnológica basada en servicios web y agentes de software sensibles al contexto.

\section{Fase 4. Validación del modelamiento propuesto a partir de casos de estudio.}

Esta última fase consiste en su aplicación a un caso de estudio relacionado con la creación de un currículo para la apertura de un nuevo programa académico de tipo universitario. Este caso hace referencia a los pasos para diseñar un currículo en una universidad. Comprende diez actividades y tres actores. Adicionalmente, la validación documental depende de los criterios de aprobación, los cuales varían de acuerdo con la institución, la fecha asociada y la personas que intervienen en la decisión que lo realiza.

\section{RESULTADOS}

\section{Gestión curricular basada en modelos de proceso de negocio}

Las actividades relevantes de la gestión curricular se centran en la planeación, evaluación, gestión pedagógica y mejoramiento. Así mismo, los actores dentro de la gestión curricular son varios, entre 
ellos se tiene al estudiante, el docente, los directivos, los asesores, la comunidad, el sector industrial, la disciplina, la familia, entre otros.

Con base en el ciclo de gestión de los procesos de negocio propuesto por Dumas et al. (2013), se propone la identificación de los procesos relacionados con la gestión curricular. La identificación consta de la definición de actividades de negocio asociadas, la identificación de roles y su relación con las actividades y la definición de una arquitectura de procesos para la gestión curricular.

La Tabla 1 presenta las actividades de negocio de la gestión curricular frente a servicios que esta ofrece. Cada una de las celdas contiene información asociada a las acciones que deben llevarse a cabo para dar cumplimiento a dichos servicios. Cabe señalar que un servicio puede ser visto como un producto, dependiendo del tipo de organización que se esté analizando.

Tabla 1. Actividades de negocio y servicios de la gestión curricular

Table 1 . Business activities and curriculum management services

\begin{tabular}{|c|c|c|c|c|}
\hline \multicolumn{5}{|c|}{ Servicios } \\
\hline $\begin{array}{c}\text { Actividad de } \\
\text { negocio }\end{array}$ & $\begin{array}{c}\text { Gestión } \\
\text { Pedagógica }\end{array}$ & $\begin{array}{l}\text { Enseñanza / } \\
\text { Aprendizaje }\end{array}$ & $\begin{array}{c}\text { Desarrollo de } \\
\text { estudiante }\end{array}$ & Pertinencia \\
\hline Planeación & $\begin{array}{l}\text {-Diseño de macro } \\
\text { currículo. } \\
\text {-Selección y ubicación } \\
\text { de recursos. } \\
\text {-Generación y } \\
\text { programación de } \\
\text { actividades. } \\
\text {-Diseño de } \\
\text { mecanismos e } \\
\text { instrumentos de } \\
\text { monitoreo. }\end{array}$ & $\begin{array}{l}\text {-Adopción de micro } \\
\text { currículos. }\end{array}$ & $\begin{array}{l}\text {-Registro de casos } \\
\text { especiales. } \\
\text {-Fomentar el ingreso } \\
\text { y la continuación. } \\
\text { - Asesoría para su } \\
\text { ingreso. }\end{array}$ & $\begin{array}{l}\text {-Informar oferta } \\
\text { educativa. } \\
\text {-Validar oferta } \\
\text { educativa. }\end{array}$ \\
\hline Aplicación & $\begin{array}{l}\text { - Análisis del uso de } \\
\text { recursos. } \\
\text {-Garantizar } \\
\text { operatividad. } \\
\text {-Seguimiento a micro } \\
\text { currículos. } \\
\text {-Recolección } \\
\text { información macro. }\end{array}$ & $\begin{array}{l}\text {-Registro de avances } \\
\text { en contenidos. } \\
\text {-Documentación de } \\
\text { experiencias. } \\
\text {-Ejecución de } \\
\text { actividades de } \\
\text { evaluación. }\end{array}$ & $\begin{array}{l}\text {-Registro de } \\
\text { asesorías } \\
\text {-Fomentar la } \\
\text { continuidad. } \\
\text {-Identificación de } \\
\text { comportamientos. }\end{array}$ & $\begin{array}{l}\text {-Recolección de } \\
\text { información del } \\
\text { entorno. } \\
\text {-Identificación de } \\
\text { patrones de } \\
\text { comportamientos. }\end{array}$ \\
\hline Evaluación & $\begin{array}{l}\text {-Alcance del } \\
\text { cronograma. } \\
\text {-Evaluación de } \\
\text { avances de } \\
\text { contenidos. } \\
\text {-Validación con } \\
\text { referentes. } \\
\text {-Análisis de } \\
\text { contenidos. } \\
\text {-Validación de } \\
\text { proceso. }\end{array}$ & $\begin{array}{l}\text {-Evaluación de } \\
\text { contenidos. } \\
\text {-Identificación de } \\
\text { fortalezas y } \\
\text { debilidades. } \\
\text {-Reporte general. } \\
\text {-Registro casos } \\
\text { especiales. } \\
\text {-Uso de los recursos. }\end{array}$ & $\begin{array}{l}\text {-Retrospectiva de } \\
\text { desarrollo. } \\
\text {-Planeación de } \\
\text { continuidad. }\end{array}$ & $\begin{array}{l}\text {-Validación de } \\
\text { actores. } \\
\text {-Análisis de } \\
\text { pertinencia e } \\
\text { impacto. }\end{array}$ \\
\hline
\end{tabular}

Fuente: elaboración propia. 
Los actores identificados para cada uno de los procesos son: Estudiante (E), Docente (D), Directivos $(\mathrm{Dr})$, Sector (S), organismos disciplinares (O) y comunidad (C). El estudiante es quien se beneficia directamente de la gestión curricular, y es por los estudiantes que se ofrece una permanente actualización. El docente apoya la formación del estudiante, brindando experiencia e información necesaria para un aprendizaje exitoso. Los directivos son quienes permiten que los currículos sean administrativa y financieramente viables. Por su parte, los organismos disciplinares están relacionados con los entes del Gobierno que regulan el diseño e implementación del currículo. A su vez, la comunidad apoya e influye en el desarrollo del estudiante. La información que cada uno de ellos brinda puede afectar su contexto y por lo tanto las decisiones que puedan tomarse durante la ejecución de proceso. La Tabla 2 identifica la participación de los actores en las actividades de negocio de la gestión curricular.

Tabla 2. Roles y actividades de negocio

Table 2. Business roles and activities

\begin{tabular}{|c|c|c|c|c|c|c|c|}
\hline & Actividad/Rol & $E$ & D & $\mathrm{Dr}$ & $\mathrm{S}$ & 0 & C \\
\hline \multirow{6}{*}{ Planeación } & Diseño macro & & $x$ & $x$ & $\mathrm{x}$ & $\mathrm{x}$ & \\
\hline & Definición de actividades pedagógicas & & $x$ & $x$ & & & \\
\hline & Selección y ubicación de recursos & & & $x$ & & $x$ & \\
\hline & Identificación y registro de casos especiales & & $x$ & $x$ & & & \\
\hline & Informar oferta & & & $x$ & & & \\
\hline & Adopción de micro-currículos & $\mathrm{x}$ & $x$ & $x$ & & & \\
\hline \multirow{8}{*}{ Aplicación } & Registro de avances & $x$ & $x$ & & & & \\
\hline & Registro de asesorías & $\mathrm{x}$ & $x$ & & & & \\
\hline & Documentación de experiencias & $x$ & $x$ & $x$ & $\mathrm{x}$ & & \\
\hline & Análisis de gestión de recursos & & & $x$ & & $\mathrm{x}$ & \\
\hline & Análisis entorno educativo & $\mathrm{x}$ & $x$ & $x$ & & & $\mathrm{x}$ \\
\hline & Seguimiento de micro-currículo & $x$ & $x$ & $x$ & $x$ & & $\mathrm{x}$ \\
\hline & Censado de información macro-curricular & & $x$ & $x$ & & & \\
\hline & Aplicación de estrategias meso-curriculares & $x$ & $x$ & & & & $\mathrm{x}$ \\
\hline \multirow{7}{*}{ Evaluación } & Evaluación de cronograma & & $x$ & $x$ & & & \\
\hline & Evaluación de avances de contenidos & & & $x$ & $x$ & & \\
\hline & Análisis de consistencia con referentes & & & $x$ & & $X$ & \\
\hline & Revisión de contenidos & & $\mathrm{x}$ & $x$ & & & \\
\hline & Análisis de pertinencia & & & $x$ & $x$ & & \\
\hline & Retrospectiva a estrategias de desarrollo & & & $x$ & & $x$ & $\mathrm{x}$ \\
\hline & Validación del proceso de gestión curricular & $\mathrm{x}$ & $\mathrm{x}$ & $\mathrm{x}$ & & & \\
\hline
\end{tabular}


Con base en la información de la Tabla 2, se origina la arquitectura de procesos propuesta para la adopción de la gestión curricular. Básicamente se busca agrupar los servicios con base en los factores incluidos en la gestión de currículo. De esta manera, se identifica la necesidad del diseño de un proceso de negocio que permita la participación de los roles definidos.

La Figura 1 presenta la arquitectura propuesta, donde cada rectángulo agrupa responsabilidades que asociadas a cada uno de los servicios. La agrupación, en este caso, se realiza a partir del análisis de la participación de cada uno de los roles en las actividades de negocio.

\begin{tabular}{|c|c|c|c|c|}
\hline & $\begin{array}{c}\text { Gestión } \\
\text { Pedagógca }\end{array}$ & $\begin{array}{l}\text { Enseñanza- } \\
\text { Aprendizaje }\end{array}$ & $\begin{array}{l}\text { Desarrollo del } \\
\text { estudiante }\end{array}$ & Entorno \\
\hline \multicolumn{5}{|l|}{ Planeación } \\
\hline \multicolumn{5}{|l|}{ Aplicación } \\
\hline Evaluación & & & & \\
\hline
\end{tabular}

Figura 1. Arquitectura de procesos para la gestión curricular

Figure 1. Process architecture for curriculum management Fuente: elaboración propia.

\section{Modelo de contexto para la gestión curricular}

Es indispensable disponer de la información relacionada con el contexto del proceso, de sus características de ejecución y del dominio, con el fin de facilitar la definición de reglas de razonamiento que soporten la toma de decisiones. Las entidades definidas para el contexto de la gestión curricular son las siguientes: recursos asociados, estructura del proceso, comportamiento del proceso, roles, organización y dominio.

En la Tabla 3 se condensan las situaciones definidas para cada una de las entidades del contexto de la gestión curricular. El valor contextual debe considerar la información dispuesta en cada uno de los procesos y sus objetos. Así mismo, las situaciones contextuales se definen teniendo en cuenta los elementos contextuales y sus valores. Esto quiere decir que, a diferencia de los eventos, las situaciones analizan la información de varios objetos, con el fin de determinar las acciones que activaron una situación específica. Con base en las entidades identificadas y las situaciones relacionadas, se propone que el contexto de un proceso de negocio facilite el razonamiento inteligente por medio de los siguientes elementos:

- Contexto de recursos: Incluye las entidades relacionadas con el consumo de recursos para la ejecución de un proceso junto con sus actividades.

- Contexto del proceso: Agrupa las entidades que permiten la representación, almacenamiento, instanciación, variación y ejecución de modelos de proceso. 
- Contexto de los participantes: Esta dimensión comprende información relacionada con las competencias, experiencia, uso del proceso y disponibilidad. Cada usuario representa una interacción con el proceso, indicando en cada interacción un posible contexto asociado.

- Contexto de la organización: Esta dimensión hace referencia a la información asociada a sus reglas de negocio; también incluye reglas de tipo externo (por ejemplo, leyes de un determinado país).

- Contexto del dominio: Comprende los conceptos relacionados con el dominio de ejecución del proceso; tiene relación directa con el ambiente de ejecución y el contexto.

- Contexto de las actividades: Esta dimensión se relaciona con la información de las actividades, su ejecución y recursos empleados.

Tabla 3. Situaciones contextuales

Table 3. Contextual situations

\begin{tabular}{|c|c|c|}
\hline Elementos & Valor contextual & Situación contextual \\
\hline \multirow{2}{*}{ Recursos asociados } & Cambios del recurso & Los cambios afectan la usabilidad de los recursos \\
\hline & Estructura del proceso & $\begin{array}{l}\text { Los formatos de los datos definidos son aplicados } \\
\text { en varios procesos }\end{array}$ \\
\hline \multirow{2}{*}{ Estructura del proceso } & Dominio & Se presenta incompatibilidad conceptual \\
\hline & Cambios & Los cambios afectan el modelo de referencia \\
\hline \multirow{2}{*}{$\begin{array}{l}\text { Comportamiento del } \\
\text { proceso }\end{array}$} & Dominio & Se presenta incompatibilidad conceptual \\
\hline & Variantes del proceso & $\begin{array}{l}\text { La regresión evaluada no es coherente con } \\
\text { resultados anteriores }\end{array}$ \\
\hline \multirow[t]{2}{*}{ Participante/Rol } & Conocimiento & $\begin{array}{l}\text { No hay disponibilidad del recurso y existe un } \\
\text { posible reemplazo }\end{array}$ \\
\hline & Funcionamiento & El sistema externo está disponible \\
\hline Organización y dominio & $\begin{array}{l}\text { Restricciones organizaciones } \\
\text { y operacionales }\end{array}$ & $\begin{array}{l}\text { Las restricciones relacionan la información de las } \\
\text { entidades contextuales. }\end{array}$ \\
\hline
\end{tabular}

Se ha diseñado un esquema de representación de procesos de negocio mediante sintaxis XPDL (XML Process Definition Language), en el que es posible asociar restricciones para su posterior procesamiento en un mecanismo de inferencia. En la Figura 2 se presentan las dimensiones contextuales que componen al contexto de la gestión curricular. Cada uno de los elementos puede ser modelado a partir de atributos que son susceptibles a cambios en su contexto, lo que conlleva que en diferentes situaciones unos atributos se impacten y en otras situaciones sean otros los atributos impactados. 


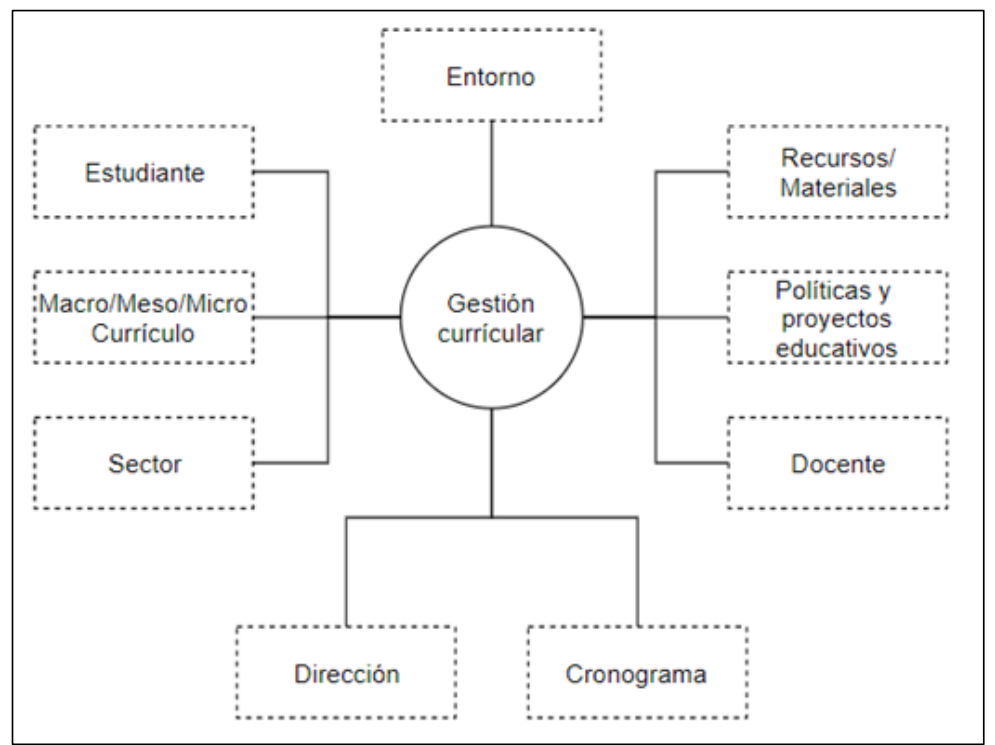

Figura 2. Dimensiones contextuales de la gestión curricular

Figure 2. Contextual dimensions of curriculum management Fuente: elaboración propia.

\section{Diseño del mecanismo de razonamiento}

En esta sección se presenta el diseño del sistema inteligente encargado del procesamiento de la información relacionada con los procesos, el procesamiento del contexto y el razonamiento de este, con el fin de tomar decisiones frente al comportamiento de las variantes del proceso que se puedan presentar (La Rosa et al., 2017).

\section{Esquema General}

El diseño comprende un sistema de representación de modelos de procesos, un sistema de razonamiento y una arquitectura tecnológica basada en servicios web y agentes de software sensibles al contexto. La Figura 3 presenta el esquema general del sistema de razonamiento. Parte de unas colecciones de modelos de proceso y pasa a traducirlas en grafos dirigidos; haciendo uso de tablas de adyacencia, de pesos y objetos complejos, se realizan actividades como comparación de procesos, identificación de flujos y aplicación de cambios.

\section{Reglas de producción}

Las reglas definen la consecuencia a partir de la activación de antecedentes. De este modo, el antecedente de la regla es la situación que se presente, y el consecuente, la acción a realizar. Las reglas tienen la siguiente forma: Si una situación se presenta y la información contextual afecta el proceso, entonces se deben validar los estados necesarios para su adaptación y registro de decisiones.

Con base en lo anterior, se proponen ocho (8) reglas de razonamiento, las cuales se organizan en cuatro grupos: niveles de información, entidades, contexto y gestión del método. La Tabla 4 consolida 
las reglas para cada uno de los grupos. La representación en expresión lógica permite la implementación de estrategias de razonamiento como en el caso del encadenamiento hacia adelante.

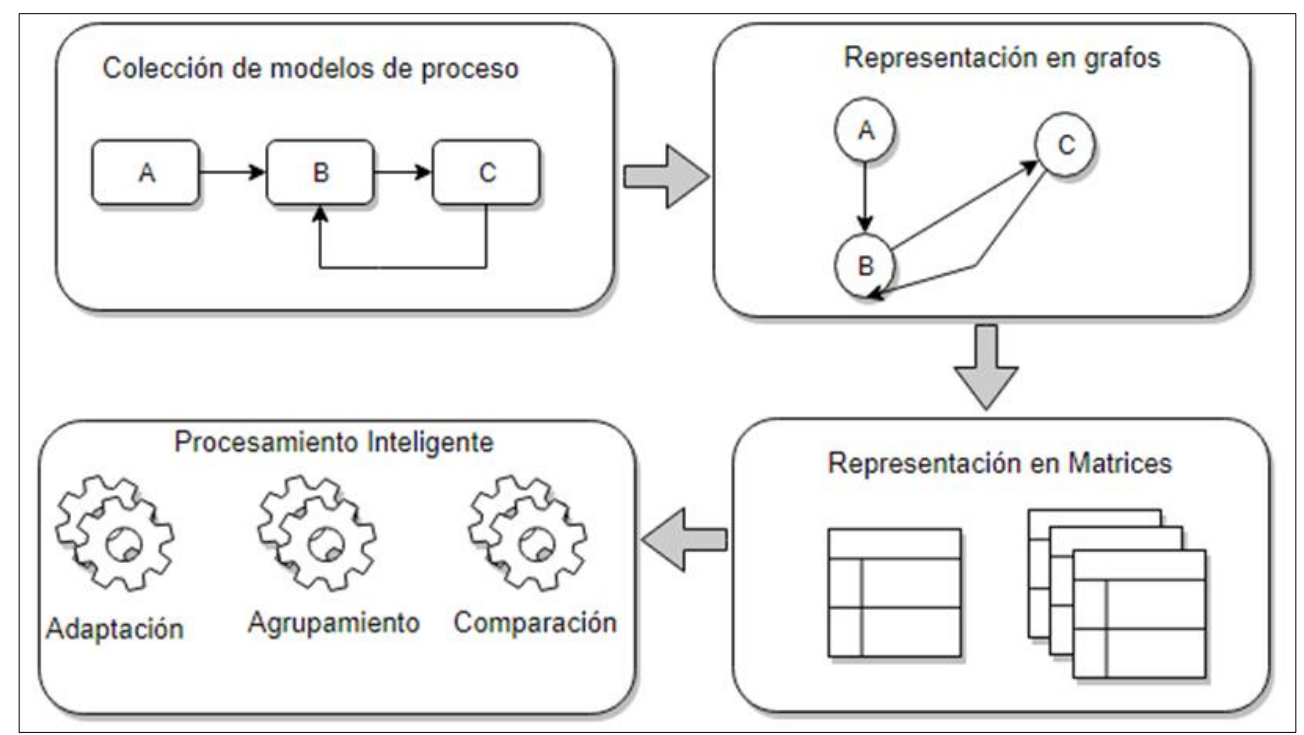

Figura 3. Estructura general del sistema de razonamiento

Figure 3. General structure of the reasoning system

Fuente: elaboración propia.

Tabla 4. Reglas de producción para el razonamiento sobre el contexto

Table 4. Context reasoning rules

\begin{tabular}{|c|c|}
\hline $\begin{array}{l}\text { Grupo de } \\
\text { reglas }\end{array}$ & Expresión lógica \\
\hline \multirow{2}{*}{$\begin{array}{l}\text { Reglas de los } \\
\text { niveles de } \\
\text { información }\end{array}$} & valida_Sit $($ Sit $[i]$, Dom, Pr $) \wedge E C t x($ ? nivel_log $) \rightarrow \operatorname{get}($ ?registro $) \wedge$ get $($ Ctx_capa $)$ \\
\hline & valida_Sit $($ Sit $[i]$, Dom,Pr $) \wedge E C t x(?$ nivel_fis $) \rightarrow \operatorname{get}(?$ registro $) \wedge$ get $($ Ctx_capa $)$ \\
\hline \multirow{2}{*}{$\begin{array}{l}\text { Reglas de las } \\
\text { entidades }\end{array}$} & valida_Sit $($ Sit $[i]$, Dom, Pr $) \wedge v_{-}$conceptual $(?$ this $) \rightarrow \operatorname{get}(?$ ent $) \wedge \operatorname{getAtr}(?$ ent $)$ \\
\hline & $\begin{aligned} \text { valida_Sit }(\operatorname{Sit}[i], \operatorname{Dom}, \operatorname{Pr}) & \wedge \operatorname{get}(? \text { ent }) \\
& \rightarrow \operatorname{validar}(? \operatorname{Dim}(\text { ent }[i])) \wedge \operatorname{sensores}(? \text { ent }, ? \operatorname{dom}) \wedge \operatorname{Ctx}(? \operatorname{dom})\end{aligned}$ \\
\hline \multirow{2}{*}{$\begin{array}{l}\text { Reglas del } \\
\text { contexto }\end{array}$} & $\begin{aligned} \text { valida_Sit }(\text { Sit }[i], \text { Dom, Pr }) & \wedge \text { get }(? \text { ent }) \\
& \rightarrow \text { afecta }(? \text { va_ctx, ? sit }) \wedge \text { aplicacion(?decision, manual })\end{aligned}$ \\
\hline & valida_Sit $($ Sit $[i]$, Dom, Pr $) \wedge \operatorname{set}(?$ dom $) \rightarrow \operatorname{validar}(? \operatorname{ct} x, ? \operatorname{pr}[i], ?$ ontologia $)$ \\
\hline \multirow{2}{*}{$\begin{array}{l}\text { Reglas del } \\
\text { método }\end{array}$} & $\begin{array}{l}\text { valida_Sit(Sit }[i], \text { Dom, } P r) \wedge \text { fase }(? \text { this }) \\
\rightarrow \text { eval }(? \text { desicion }(\text { ¿this })) \wedge \text { set_reporte }(? \text { fase }[i])\end{array}$ \\
\hline & $\begin{aligned}\text { valida_Sit(Sit) }) & \wedge \text { alertas }(? \text { ctx }, \text { dom }) \\
& \rightarrow \text { registro_historico }(? \text { proceso }) \wedge \text { adaptacion }(\text { decisiones }[])\end{aligned}$ \\
\hline
\end{tabular}


La Figura 4 presenta el algoritmo definido para el razonamiento inteligente. Se compone de dos subprogramas para la propagación de cambios y para el agrupamiento. El algoritmo trabaja con la colección de modelos y con las reglas definidas e inicia cuando se hace una representación en grafos a cada uno de los modelos de proceso, para así generar matrices de incidencia y adyacencia y poder continuar con la definición de estados factibles. Si el flujo de los procesos es igual, entonces se propagan los cambios, de lo contrario, se debe realizar un agrupamiento para definir un solo modelo de propagación.

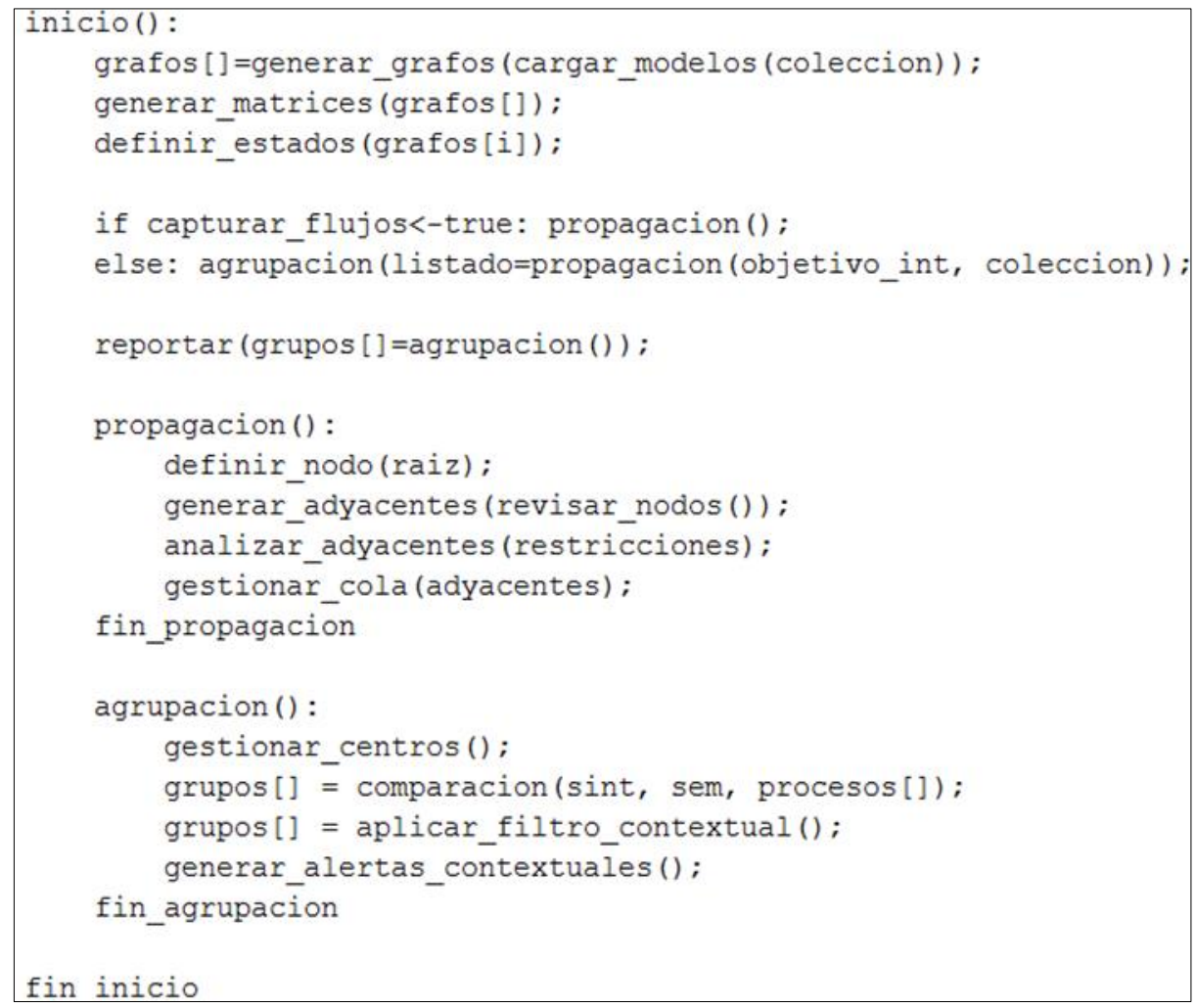

Figura 4. Algoritmo general del sistema de razonamiento

Figure 4. General algorithm of the reasoning system Fuente: elaboración propia.

En el procedimiento de propagación se emplean técnicas de verificación de restricciones para poder valorar las opciones de solución con previa aceptación. Por su parte, el agrupamiento como un filtro de las a las variantes previamente seleccionadas.

\section{Validación del mecanismo de razonamiento a partir de un caso de estudio}

La validación del modelo de contexto consiste en su aplicación a un caso de estudio relacionado con la creación de un currículo para la apertura de un nuevo programa académico de tipo universitario. La Figura 5 presenta el diagrama general del proceso: creación de currículo. El proceso hace referencia a los pasos para diseñar un currículo en una universidad. Comprende diez actividades y tres actores. Las variables prioritarias que se deben gestionar por parte del contexto son la validación documental y la aprobación del currículo. 


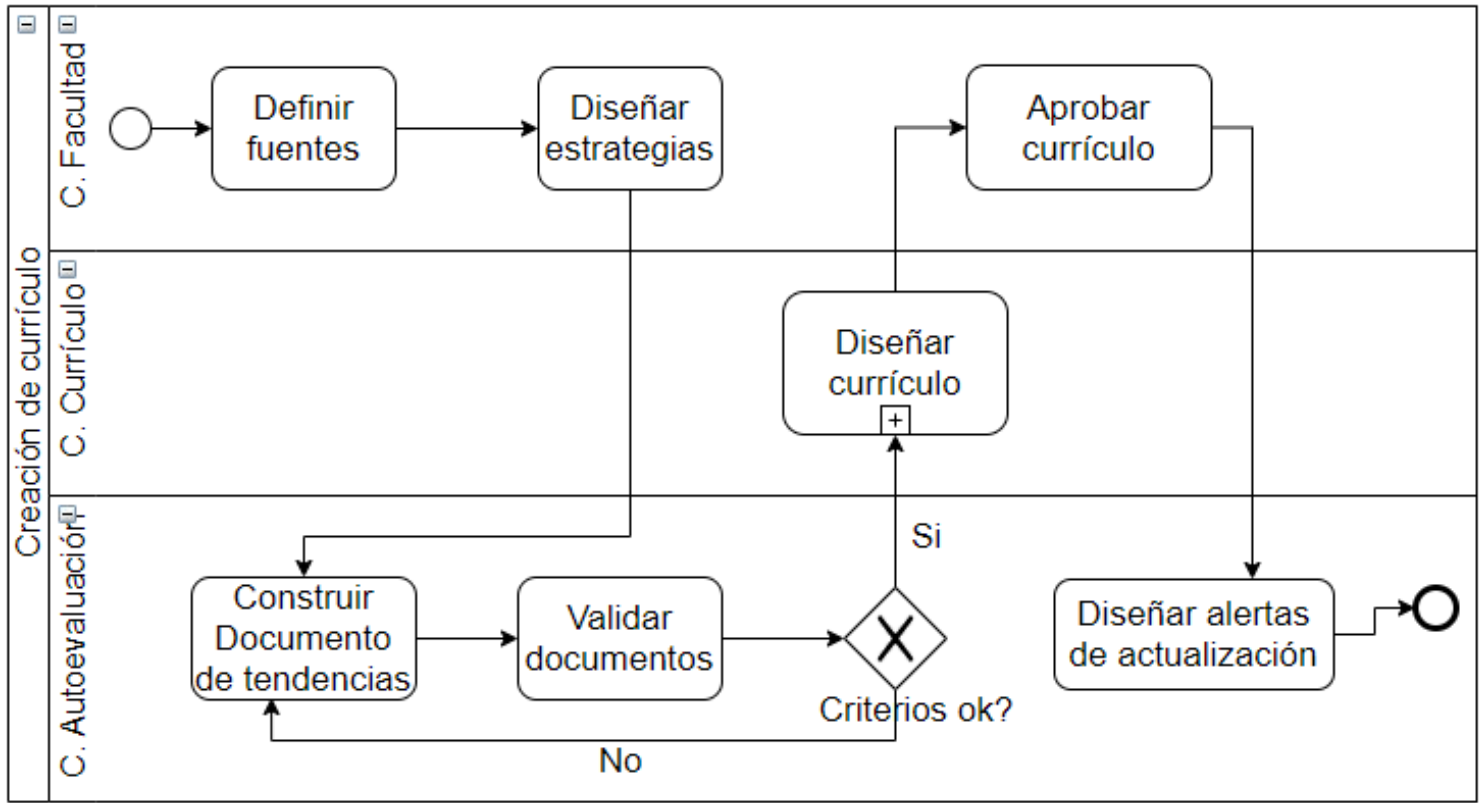

Figura 5. Proceso de negocio «creación de currículo»

Figure 5. "Curriculum creation" business process

Fuente: elaboración propia.

La validación documental depende de los criterios de aprobación, los cuales varían de acuerdo con la institución, la fecha asociada y la personas que intervienen en la decisión que lo realiza. En términos de personal, las competencias pueden ser reemplazadas con base en la experiencia de sus integrantes. Por su parte, la aprobación del currículo depende de la competencia de sus individuos, ya que, en algunos casos, debido a sus sistemas de conformación, se deben asignar asesores con experiencia.

\section{Generación del grafo representativo}

Para el análisis del proceso desde un punto de vista del contexto, se procede a realizar una transformación del modelo en un grafo representativo. Dicho grafo es de tipo dirigido, y actividades, estructuras de control y puntos de inicio/fin se consideran vértices para el grafo. En la Figura 6 se presenta el grafo generado del modelo del caso de estudio del diseño de un currículo.

\section{Razonamiento sobre el contexto}

Una vez generado el grafo representativo y definidas las matrices de adyacencia e incidencia, se procede a sensibilizar el modelo para la detección de situaciones. Dicha sensibilización consiste en la definición de sensores virtuales de captura de información; así mismo se hace uso de técnicas de similitud para la comparación de los modelos de proceso con otros modelos dentro de la misma organización, esto con el fin de identificar patrones similares de comportamiento y reacción a situaciones. 


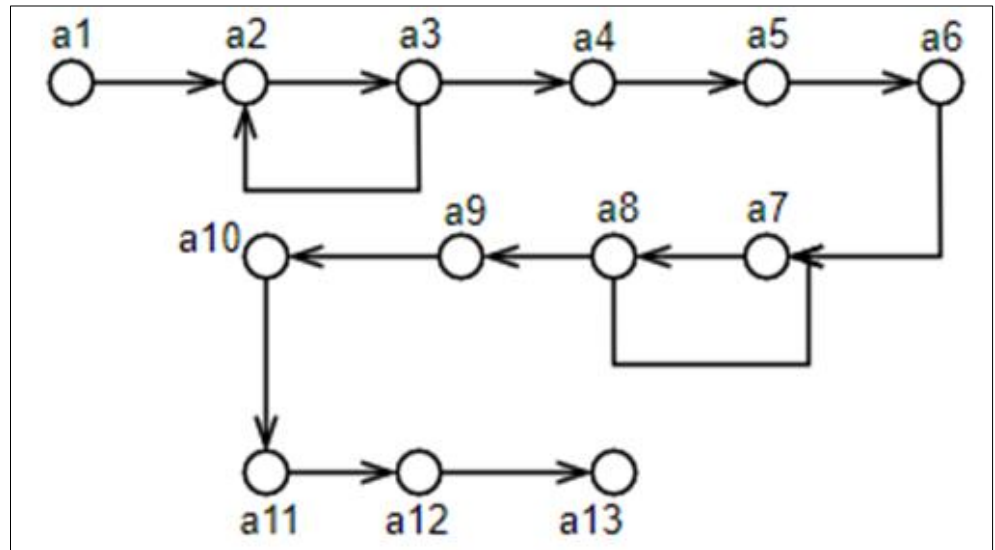

Figura 6. Grafo representativo del caso de estudio

Figure 6. Graphical representation of the case study Fuente: elaboración propia.

\section{DISCUSIÓN}

En síntesis, para la validación del método se definieron métricas de evaluación de acuerdo con las capacidades del sistema, donde se tiene en cuenta la gestión y representación de procesos, la búsqueda y recuperación, la integración y ejecución y el razonamiento en general. Las métricas definidas son:

- Precisión: indica el número elementos relevantes a partir del conjunto de los elementos recuperados.

- Recuperación: determina el número de elementos relevantes que son recuperados. Junto con la Precisión se calcula el valor de la métrica F, la cual sirve para dar un grado de proximidad entre la precisión y recuperación.

- Alineación: capacidad de identificar características similares y diferencias entre dos procesos. Las diferencias se marcan como caminos alternos en el grafo. Adicionalmente, los pesos de dichos caminos afectan la alineación. Las etiquetas lingüísticas asociadas para su valoración son: alta, media y baja.

- Correctitud: determina si los datos, pesos y grados de similitud en todo el proceso son coherentes con valores definidos para el método. Wu et al., (2016) sugieren que la correctitud se asocie al cumplimiento de las reglas organizacionales y operacionales definidas.

- Tiempo razonamiento: indica el tiempo delta de ejecución del mecanismo de razonamiento. Dicho criterio puede ser utilizado para el diseño de técnicas de mejoramiento de rendimiento.

En la Tabla 5 se presenta la evaluación del modelo para el caso de estudio "creación de currículo" para un programa nuevo. Se generaron conjuntos de variantes del proceso, se definieron variables para el contexto, así como los valores asociados; posteriormente, se realizó una ejecución de los procesos para así capturar datos de comportamiento. 
Tabla 5. Evaluación del caso de estudio

Table 5. Case study evaluation

\begin{tabular}{ccc}
\hline Métrica & Valor & Descripción \\
\hline Precisión & 0,92 & $\begin{array}{c}\text { Métrica basada en grado de proximidad de } \\
\text { los modelos de procesos. }\end{array}$ \\
\hline Recuperación & 0,89 & $\begin{array}{c}\text { El sistema determina la relación de los } \\
\text { modelos de procesos. }\end{array}$ \\
\hline Alineación & Alta & El sistema identifica características similares \\
y diferentes.
\end{tabular}

A partir de los resultados obtenidos, y después de implementar y validar el modelo de contexto propuesto en el caso de estudio, se puede observar que:

- El modelo genera resultados apropiados para la gestión de modelos de proceso, así como para la generación de grafos representativos.

- El modelo se soporta apropiadamente con la búsqueda y recuperación de modelos de proceso, generando una lista de los más priorizados.

- El modelo depende del diseño del caso de estudio, ya que comprende un gran número de instancias.

- Para complementar las soluciones que mejoren el rendimiento, se pueden aplicar mecanismos de razonamiento que permitan realizar una búsqueda de rutas de solución. Con ello, se podría trabajar con históricos para mejorar la recuperación y procesamiento de las instancias de los modelos de proceso.

Como principal aporte de la investigación, se tiene la representación de la gestión curricular desde el punto de vista de los procesos de negocio sensibles al contexto, lo que permite obtener, de una manera estructurada, información adicional del entorno de ejecución del proceso y las situaciones que se presenten. A partir de ello, es posible diseñar reglas de razonamiento que permitan tener en cuenta el contexto para la toma de decisiones.

Otras aproximaciones investigativas a la temática consideran el modelamiento de las variables de la gestión curricular en términos de la estructura de información orientada a la definición de modelos de datos (Cottrell et al., 2004; Watson et al., 2007). Por su parte, lo propuesto en este artículo considera que los datos son susceptibles a la dinámica del entorno de ejecución del dominio, siendo este un componente diferencial que puede abrir la posibilidad de investigaciones futuras que validen el modelo propuesto en otras instituciones de educación superior.

Adicionalmente, el modelo de gestión curricular propuesto se caracteriza por ser generalizado, lo que quiere decir que puede ser aplicado en distintos dominios de ejecución; por ejemplo, la producción de alimentos, la admisión de estudiantes en una universidad o el flujo editorial de una revista 
científica. Lo anterior evidencia su replicabilidad y posibilidades de aplicación en otros ámbitos de interés.

\section{CONCLUSIONES}

La inclusión de un modelo de contexto que represente las situaciones que identifican entidades, permite la captura de información adicional que puede ser aprovechada por mecanismos de razonamiento, los cuales contienen datos propios del dominio y ambiente de ejecución. Asimismo, el modelo de contexto propuesto para la gestión del currículo está basado en información contextual de entidades, que a su vez componen las situaciones contextuales propias del proceso. Así pues, el modelo facilita el razonamiento basado en inferencias sobre un proceso específico.

El mecanismo de razonamiento propuesto para el procesamiento del contexto mejora la comprensión de los procesos de negocio, sin embargo, el mecanismo presenta algunas limitaciones que son posibles líneas de investigación dentro del área, estas son: mejoramiento de las estructuras de datos de los grafos representativos y desarrollo de protocolos de interacción entre el proceso y el contexto asociado.

Por último y no menos importante, es necesario explorar el concepto de variantes de proceso. Este concepto busca la representación de modelos de proceso de las distintas versiones de un mismo proceso. Las variantes se generan a partir de un modelo de referencia. Es así que, cualquier cambio que se produzca en un modelo de referencia, debe ser propagado a las variantes. Con base en lo anterior, se requiere la definición de modelos de referencia que faciliten la propagación de cambios sobre colecciones de procesos con el fin de realizar actividades de mantenimiento y actualización.

\section{REFERENCIAS}

Barrón Tirado, M. C.; Díaz Barriga, F. (2016). Curriculum Management and the Role of Curriculum Actors. TCI Transnational Curriculum Inquiry, v. 13, n. 2, 13-33. URL

Benedict, T.; Bilodeau, N.; Vitkus, P.; Powell, E.; Morris, D.; Scarsig, M.; Lee, D.; Field, G.; Lohr, T.; Saxena, R. (2013). BPM CBOK Version 3.0: guide to the business process management common body of knowledge. ABPMP International/Createspace.

Cottrell, S.; Linger, B.; Shumway, J. (2004). Using information contained in the curriculum management information tool (CurrMIT) to capture opportunities for student learning and development. Medical Teacher, v. 26, n. 5, 423-427. https://doi.org/10.1080/0142159042000218650

Decreto 230 de 2002 [Ministerio de Educación Nacional de Colombia]. Por el cual se dictan normas en materia de currículo, evaluación y promoción de los educandos y evaluación institucional. 11 de febrero de 2002. 
Dey, A. K. (2001). Understanding and using context. Personal and ubiquitous computing, v. 5, n. 1, 47. https://doi.org/10.1007/s007790170019

Dumas, M.; La Rosa, M.; Mendling, J.; Reijers H. A. (2013). Fundamentals of Business Process Management, Springer. https://doi.org/10.1007/978-3-662-56509-4

Giraldo Plaza, J.; Ovalle Carranza, D.; Santoro, F. (2019). Sistema para la Integración de procesos de Negocio basado en situaciones contextuales. Caso estudio: Admisión Universitaria. Scientia et Technica, v. 23, n. 4, 519-527.

González Jiménez, C. (2015). Diagnosis on curriculum management and administration headquarters interuniversity of Alajuela, National University Costa Rica-Calidad en la Educación Superior, v. 6, n. 2, 64-86. URL

Jacobs, J.; Salas, A., Cameron, T.; Naguwa, G.; Kasuya, R. (2005). Implementing an online curriculum management database in a problem-based learning curriculum. Academic medicine: journal of the Association of American Medical Colleges, v. 80, n. 9, 840-846. https://doi.org/10.1097/00001888-200509000-00011

Kahloun, F.; Channouchi, S. A. (2016). Quality criteria and metrics for business process models in higher education domain: case of a tracking of curriculum offers process. Procedia Computer Science, v. 100, 1016-1023. https://doi.org/10.1016/j.procs.2016.09.274

Kim, S. N. (2005). The developmental directions and tasks of the School Based Curriculum Management system in Korea. Asia Pacific Education Review, v. 6, n. 1, 41-49. URL

Krumm, J. (2010). Ubiquitous computing fundamentals. $\underline{\text { URL }}$

La Rosa, M.; Van der Aalst, W. M. P.; Dumas, M.; Milani, F. P. (2017). Business process variability modeling: A survey. ACM Computing Surveys, v. 50, n. 1, 2. https://doi.org/10.1145/3041957

Maia, I. B.; Pacheco, J. A. (2019). Curriculum management based on results and standards and their effects on institutional evaluation. Laplage em Revista, v. 5, 18-30. URL

Makura, O. y Makura, A. H. (2012). Rethinking the definition and value of the curriculum concept: The Zimbabwe experience. The Anthropologist, v. 14, n. 6, 509-515. https://doi.org/10.1080/09720073.2012.11891276

Morales Martínez, M. E.; Preciado Duarte, N. Y.; Nader, V. S.; Hernández, C. P. (2017). La gestión curricular; procesos y tendencias. una revisión documental. Especialización en docencia universitaria. Fundación Universitaria de ciencias de la salud. Bogotá.

Ploesser, K.; Peleg, M.; Soffer, P.; Rosemann, M.; Recker, J. C. (2009). Learning from context to improve business processes. BPTrends, v. 6, n. 1, 1-7. URL 
Rosa, J. H.; Barbosa, J. L. V.; Kich, M.; Brito, L. (2015). A multi-temporal context-aware system for competences management. International Journal of Artificial Intelligence in Education, v. 25, n. 4, 455-492. https://doi.org/10.1007/s40593-015-0047-y

Saidani, O.; Nurcan, S. (2009, April). Context-awareness for adequate business process modelling. In 2009 Third International Conference on Research Challenges in Information Science, Fez, Morocco. https://doi.org/10.1109/RCIS.2009.5089281

Salgado Bustamante, M.; Medel Toro, J. (2015). Gestión curricular al servicio de aprendizajes significativos. [Tesis de Maestría en Liderazgo y Gestión de Establecimientos Educacionales, Universidad del Bío Bío]. URL

Strîmbei, C.; Dospinescu, O.; Strainu, R. M.; Nistor, A. (2016). The BPMN Approach of the University Information Systems. Ecoforum Journal, v. 5, n. 2. URL

Svensson, C.; Hvolby, H. H. (2012). Establishing a business process reference model for Universities. Procedia Technology, v. 5, 635-642. https://doi.org/10.1016/j.protcy.2012.09.070

Viciana, J.; Mayorga-Vega, D. (2016). Innovative teaching units applied to Physical Educationchanging the curriculum management for authentic outcomes. Kinesiology, v. 48, n. 1, 142-152. https://doi.org/10.26582/k.48.1.1

Watson, E. G.; Moloney, P. J.; Toohey, S. M.; Hughes, C. S.; Mobbs, S. L.; Leeper, J. B.; McNeil, H. P. (2007). Development of eMed: a comprehensive, modular curriculum-management system. Academic medicine: journal of the Association of American Medical Colleges, v. 82, n. 4, 351360. https://doi.org/10.1097/ACM.0b013e3180334d41

Weske, M. (2019). Business Process Management. Concepts, Languages, Architectures. https://doi.org/10.1007/978-3-662-59432-2

Wu, B.; Lin, R.; Wang, P.; Chen, J. (2016). Dynamic Business Process Generation and Verification. In 2016 IEEE International Conference on Services Computing (SCC). San Francisco. https://doi.org/10.1109/SCC.2016.118

Zimmer, W. K.; Keiper, P. (2020). Redesigning curriculum at the higher education level: challenges and successes within a sport management program. Educational Action Research. https://doi.org/10.1080/09650792.2020.1727348 